Fragen ${ }_{n}$ über die Uugerechtigkeit der mittelalterlichen ordentlichen Steuern" und „über das Verhältniss der Höhe derselben zu den ausserordentlichen Steuern" in dem Abschnitte uber das Steuerwesen in Wälschtirol. Erst mit Hilfe des Inhaltsverzeichnisses kann man sich da Klarheit über die Disponirung des Stoffes schaffen.

Alle erwähnten Mängel, wie sie ja bei Erstlingsarbeiten in dem Drange nach möglichst rascher Fertigstellung derselben vorzukommen pflegen, namentlich auch die Erscheinung, dass das Material stellenweise mehr aneinander gereiht, als nach rechtshistorischen $\mathrm{Ge}$ sichtspunkten verarbeitet wurde, und dass die einschlägige Litteratur nicht immer ausreichend herangezogen und in kritischer Weise besprochen wurde, hindern jedoch nicht, dem Verfasser für seine selbständige, fleissige Arbeit Dank zu wissen ${ }^{1}$, und so darf ich am Schlusse der Erwartung Ausdruck geben, dass Kogler, durch weitere Forschungen entsprechend geschult, die österreichische Rechtsgeschichte noch mit so mancher schönen Untersuchung erfreven wird.

Innsbruck.

A. v. Wretschko.

\title{
Dr. Max van Vleuten, Die Grunddienstbarkeiten nach
} altwestnordischem Rechte. Fine rechtsgeschichtliche Abhandlung. $8^{\circ}$ (VIII und $183 \mathrm{~S}$.). München 1902, Theodor Ackermann.

Die vorbezeichnete Schrift giebt ein anschauliches Bild der reichen und eigenartigen Gestaltung, die im norwegischen und isländischen Rechte des Mittelalters die Grunddienstbarkeiten erhalten haben. Vornehmlich bestrebt, die Quellen selbst zum Leser sprechen zu lassen, verabsäumt der Verfasser doch nicht, den allgemeinen Rechtsgedanken nachzugehen, die aus der zusammenfassenden Betrachtung der einzelnen Zeugnisse zu gewinnen sind. Sie würden noch klarer hervortreten, wenn der Verfasser sich durehgängig auf die Behandlung seines Themas beschränkt hätte. Die Freude an dem Reichthum des Quellenmaterials mag ihn dazu verführt haben, auch solche Fragen eingehend zu erörtern, die für den von ihm behandelten Gegenstand nur mittelbar von Bedeutung sind. So leitet er die Betrachtung der für Island wichtigen Strandrechtsgerechtigkeit mit einer mehr als 20 Seiten füllenden Darstellung des Strandrechts ein (S. 75-97), der dann auf nicht mehr als 5 Seiten die Behandlung der Strandrechtsdienstbarkeit selbst folgt. Noch grösser ist das Missverhältniss, wenn nachher

1) Diese Habilitationsschrift hat in wissenschaftlichen Kreisen, wie die bisher erschienenen Kritiken zeigen, eine günstige Aufnalume gefunden. Ausser dem schon erwähnten ausführlichen Referate v. Myrbach's liegen mir noch folgende Besprechungen vor: Zeitschrift für Volkswirthschaft, Sozialpolitik und Verwaltung Bd. XI. S. 326 ff. (v. In a ma), Historische Vierteljahresschrift V. Jahrgang S. 566 ff. (v. Voltelini), Mittheilungen des Instituts für österreichische Geschichtsforschung Bd. XXIII. S. 683 ff. (Bittner) und Tridentinum Ann. IV. S. $473 \mathrm{ff}$. (Menestrina).

Zeitschrift fïr Rechtsgeschichte. XXIII. Germ. Abth. 
(S. 149-156) das norwegische Strandrecht vorgeführt und daran schliesslich die Bemerkung geknüpft wird, dass wir das Strandrecht als Dienstbarkeit in den norwegischen Quellen in keinerlei Form finden. Entsprechendes ist äber die Behandlung von Jagd und Fischerei nach den beiden Rechten zu sagen. Hier werden zunächst „die Rechtsverhältnisse von Jagd und Fischerei im allgemeinen" auf 34 Seiten dargestellt, während der den Gegenstand einer Grunddienstbarkeit bildenden Jagdund Fischereigerechtigkeit im Ganzen 6 Seiten gewidmet sind. Dadurch, dass der Verfasser (s. namentlich S. 14 ff.) die Dienstbarkeiten als Eigenthumsbestandtheile betrachtet, rechtfertigt sich sein Verfahren keineswegs. Er hätte überhaupt besser gethan, in jedem der beiden Abschnitte, in welche sich seine Abhandlung nach den zwei besprochenen Rechten gliedert, die allgemeinen Grundsätze nicht an die Spitze, sondern an den Schluss zu stellen. Das entspräche der Beschaffenheit der Quellen und würde zum Mindesten den Vortheil der Vermeidung von Wiederholungen mit sich gebracht haben.

Aus der Zahl dieser allgemeinen Grundsätze muss ich, um Einspruch zu erheben, hier erwähuen, was der Verfasser (S. 36 ff.) über die Entstebung von Dienstbarkeiten durch Ersitzung nach isländischem Rechte sagt. Er führt sechs Urkunden des Diplomatar. Island. an, aus denen mit Sicherheit hervorgehe, „dass es eine Ersitzung der Dienstbarkeit gab und dass ihre Voraussetzung: Ablauf einer längeren Frist, Ununterbrochenheit der Ausübung und Unangefochtenheit war". Neben der Ersitzung scheine aber, worauf wenigstens die letzten der angeführten Quellenstellen hindeuteten, auch das Institut der unvordenklichen Verjährung bestanden zu haben. Diese Behauptungen des Verfassers fordern nach verschiedenen Richtungen zum Widerspruche heraus. Unzulässig ist schon die Verwerthung der in Rede stehenden Urkunden als Zeugnisse für isländisches Recht schlechthin. Sie stammen aus den Jahren 1325 bis 1447 und durften daher nicht, wie geschehen, auf einer Linie mit den Quellen der Freistaatszeit genannt werden. Wenn sie wirklich für die Ersitzung von Dienstbarkeiten Zeugniss ablegten, wäre diese damit noch nicht als isländischen Ursprungs erwiesen. Indessen bedarf dies keiner weiteren Untersuchung, weil in Wahrheit keine einzige der Urkunden von einer Ersitzung spricht. Sie sind insgesammt ausgestellt über die Aussagen von Zeugen, die für längere oder kürzere Zeit das Bestehen von Grunddienstbarkeiten bekunden, niemals aber von einem anderen Rechtszustande gehört haben. ${ }^{1}$ ) Ueber den Entstehungsgrund der durch sie bezeugten Dienstbarkeiten lässt sich aus ihnen nichts entnehmen.

Wie angedeutet, liegt das Schwergewicht der uns beschäftigenden Schrift in der Vorführung der ihren Gegenstand betreffenden Quellen-

1) Auch in Dipl. Isl. II nr. 484 bekunden die Zeugen nicht, wie die Uebersetzung des Verfassers (S. 38) anzunehmen scheint, sie wüssten, dass die Berechtigten ihre Dienstbarkeit (nur) 39 Winter ausgeübt bätten, sondern sie könnten sich einer so langen Ausübung erinnern (obne dass ein längeres Bestehen dadurch ausgeschlossen würde). 
zeugnisse. Der Verfasser theilt sie in grosser Zahl im Texte und in Uebersetzung mit. Die Textbehandlung anlangend bemerkt er (S. 2), die Orthographie der benutzten Quellen sei "peinlich genau" beibehalten worden, „um es so dem fachkundigen Leser zu ermöglichen, bei zweifelhaften Stellen die Uebersetzungen einer Nachprüfung zu unterziehen". Dass für diesen Zweck jenes Mittel allgemein erforderlich oder auch nur empfehlenswerth wäre, darf bezweifelt werden. Sicher aber ist, dass der Verfasser keineswegs peinlich genau bei der Wiedergabe der Texte verfahren ist. Hierron kann sich jedermanu durch Vergleichung ohne Mühe überzeugen. An dieser Stelle sei nur zweierlei erwähnt. Die in den Urkunden gewöhnliche Abbreviatur 'forde' (für fornæmde u. dgl.) giebt der Verfasser mehrfach durch das sinnlose 'forde' wieder. Die zur Bezeichnung von Doppelbuchstaben dienenden Majuskeln der Graugansstellen ersetzt er durch einfache Minuskeln und gelangt so zu Formen, wie anars, brenir, fer, legia, logard U. s. w.

Dass der Verfasser den Quellenstellen durchweg Uebersetzungen beigiebt, ist gewiss nur zu billigen. Jakob Grimm hat es freilich abgelehnt, so zu verfahren, weil, wem es ernstlich zu thun sei um das Studium des deutschen Rechts, für den auch die Erlernung unserer Sprachdialekte nicht Hinderniss sein könne, sondern Anreizung (Rechtsalterthümer* I S. XIII). Aber so vornehm dies gedacht ist - der Sache hat es geschadet. Die Bedeutung der skandinavischen Quellen für jeden Zweig der germanischen Rechtsgeschichte wäre wohl früher in weiteren Kreisen erkannt worden, wenn nicht viele Leser zumal auch der Rechtsalterthümer an den nur im Urtexte gegebenen Quellenbelegen achtlos vorüberzugehen vermocht hätten. Freilich muss der sprachunkundige Leser sich auf die Richtigkeit der Uebersetzung verlassen können. Eben in dieser Beziehung lässt aber die Arbeit des Verfassers zu wünschen übrig. Zum Beweise sollen hier nur einige von denjenigen Uebersetzungsfehlern angeführt werden, die erheblichere, rechtsgeschichtliche Irrthümer nach sich gezogen haben.

Zu Gunsten der Ansicht, dass es sich bei dem Strandrechte der Graugans um einen sich von Rechts wegen vollziehenden Eigenthumserwerb handle, beruft sich der Verfasser (S. $85 \mathrm{f}$.) namentlich auf den Eingang des c. 209 der Kgsbk (um reka): hverr madr a reka fyrir lande sino viđar oc sela oc hvala oc fisca. Er meint, reki könne hier nicht das Recht auf die angetriebenen Gegenstände, sondern nur diese selbst bezeichnen, weil dahinter die Apposition viđar u. s. w. stehe. Das Argument (wenn auch nicht die Behauptung, die es stïtzen soll) ist unzutreffend; denn, wie schon die Form 'viđar' zeigt, handelt es sich um Genitive, nicht um Appositionen.

In Frpl. XIII 10 heisst es von denen, die eine Wegegerechtigkeit dem sie bestreitenden Grundbesitzer gegenüber als bestehend erwiesen haben: " pá sculu peir pann veg hafa. en ef peir standa fyrir. pá eru peir allir útlagir hvat sem peim verđr veitt. en hinir helgir er til sökja ${ }^{a}$. Der Verfasser übersetzt (S. 125): ${ }_{n}$ Dann sollen sie diesen Weg 
haben, und die dagegen sind, die werden alle bussfällig, wenn sie überführt werden und jene geheiligt, die helfen ${ }^{*}$. In Wahrheit ist bestimmt, dass die den Berechtigten sich in den Weg Stellenden keinen Rechtsschutz geniessen in Ansehung alles dessen, was ihnen dabei zugefügt wird, während die Angreifer geschützt sind.

Missverstanden hat der Verfasser (S. 162f.) die Vorschriften von Frpl. XIV 11 über die gesetzliche und die gewillkürte Friedheiligung der Seehundsplätze. Indem er die Worte „pess á milli“ fälschlich durch "während dieser Zeit" wiedergiebt, verkennt er, dass sie umgekehrt den ausserhalb des vorher abgegrenzten liegenden Zeitraum bezeichnen. Die richtige Uebersetzung findet sich bei Brandt Forelæsninger I 269.

Der zu manchen Zweifeln Anlass gebende' ${ }^{1}$, zweite Theil von Gpl. 94 kann keinesfalls so verstanden werden, wie dies seitens des Verfassers (S. 165f.) geschieht. Davon, dass „der Bär selbst ausbricht und dann andere auf ihn Jagd machen", ist in der Stelle schlechterdings nicht die Rede.

Wenn bei der Heringsfischerei ${ }^{2}$ ) durch Strom oder Sturm Netze in einander verwickelt worden sind und ohne Beschädigung nicht wieder getrennt werden können, soll nach MLL. VII 51, wie der Verfasser (S. 178) meint, ,der den Schaden tragen, der abwesend war; sind beide anwesend, so trägt jeder die Hälfte, ausser wenn der Schaden durch die Gewalt des einen verursacht ist". Abgesehen davon, dass der Plural völd nicht Gewalt, sondern Veranlassung (hier Verschulden) bedeutet, beruht die vom Verfasser unterstellte Ersatzpflicht des Abwesenden auf unrichtiger Uebersetzung. Es heisst im Gesetze: pá bœte skađa bann, er af verđr nema báđir sè hjá, „dann bessere man den Schaden, der daraus entsteht, ausser wenn beide anwesend sind". Die Person des Ersatzpflichtigen wird sich nach allgemeinen Grundsätzen bestimmen. ${ }^{3}$ ) Dass aber gerade der zur Zeit der Entstehung des Schadens Abwesende ihn stets hätte tragen sollen, würde eine grobe Ungerechtigkeit bedeutet haben.

Ist nach dem Bemerkten an der Arbeit des Verfassers manches auszusetzen, so wird es $\mathrm{ihm}$ doch als Verdienst angerechnet werden müssen, dass er die rechts- und wirthschaftsgeschichtlich wichtige Materie der Grunddienstbarkeiten für das westnordische Recht des Mittelalters zum Gegenstande einer selbständigen, zumal das urkundliche Material mehr als bisher berücksichtigenden Untersuchung gemacht hat.

Kiel.

Max Pappenheim.

1) Vgl. Brandt I 260, Hertzberg, Glossar s. v. vida f. - ${ }^{2}$ ) Die für sie geltenden Vorschriften sind wegen der Eigenart der thatsüchlichen Verhältnisse durchaus nicht mit dem Verfasser (S. 175) „unbedenklich auch auf die andere Seefischerei auszudehnen". - s) Während Chr. IV N. L. VI 48 noch bestimmt "bode den skaden, det aff kommer", schreibt Chr. V N.L. V 11, 23 vor: betale den skaden, som forvolder det. Wine sachliche Aenderung war hiermit nicht gegeben. Vgl. auch Forarbeiderne til Kong Christian den Femtes norske Lov. . udg. af N. Prebensen og Hj. S mith S. 459, 639. 